\title{
Efficient Generation of Parallel Quasirandom Faure Sequences Via Scrambling
}

\author{
Hongmei $\mathrm{Chi}^{1,2}$ and Michael Mascagni ${ }^{1,3}$ \\ ${ }^{1}$ School of Computational Science, Florida State University, \\ Tallahassee, FL 32306-4120, USA \\ chi@scs.fsu.edu \\ ${ }^{2}$ Department of Computer and Information Sciences, Florida A\& M University, \\ Tallahassee, FL 32307-5100, USA \\ ${ }^{3}$ Department of Computer Science, Florida State University, \\ Tallahassee, FL 32306-4530, USA
}

\begin{abstract}
Much of the recent work on parallelizing quasi-Monte Carlo methods has been aimed at splitting a quasirandom sequence into many subsequences which are then used independently on the various parallel processes. This method works well for the parallelization of pseudorandom numbers, but due to the nature of quality in quasirandom numbers, this technique has many drawbacks. In contrast, this paper proposes an alternative approach for generating parallel quasirandom sequences via scrambling. The exact meaning of the digit scrambling we use depends on the mathematical details of the quasirandom number sequence's method of generation. The Faure sequence is scramble by modifying the generator matrices in the definition. Thus, we not only obtain the expected near-perfect speedup of the naturally parallel Monte Carlo methods, but the errors in the parallel computation is even smaller than if the computation were done with the same quantity of quasirandom numbers using the original Faure sequence.
\end{abstract}

Keywords: Parallel computing, Faure sequence, quasi-Monte Carlo, scrambling, optimal sequences.

\section{Introduction}

One major advantage of Monte Carlo methods is that they are usually very easy to parallelize; this leads us to all Monte Carlo methods "naturally parallel" algorithms. This is, in principal, also true of quasi-Monte Carlo (QMC) methods. As with ordinary Monte Carlo, QMC applications have high degrees of parallelism, can tolerate large latencies, and usually require considerable computational effort, making them extremely well suited for parallel, distributed, and even Grid-based computational environments. Parallel computations using QMC require a source of quasirandom sequences, which are distributed among the individual parallel processes. In these environments, a large QMC problem can be broken up into many small subproblems. These subproblems are then scheduled 
on the parallel, distributed, or Grid-based environment. In a more traditional instantiation, these environments are usually a workstation cluster connected by a local-area network, where the computational workload is carefully distributed. Recently, peer-to-peer and Grid-based computing, the cooperative use of geographically distributed resources unified to act as a single powerful computer, has been investigated as an appropriate computational environment for Monte Carlo applications 910]. QMC methods can significantly increase the accuracy of the likelihood-estimated over regular MC [7. In addition, QMC can improve the convergence rate of traditional Markov chain Monte Carlo [16. This paper explores an approach to generating parallel quasirandom sequences for parallel, distributed, and Grid-based computing.

Like pseudorandom numbers, quasirandom sequences are deterministically generated, but, in contrast, are constructed to be highly uniformly distributed. The high level of uniformity is a global property of these sequences, and something as innocent sounding as the deletion of a single quasirandom point from a sequence can harm its uniformity quite drastically. The measure of uniformity used traditionally in quasirandom number is the star-discrepancy. The reason for this is the Koksma-Hlawka inequality. This is the fundamental result in QMC theory, and motivates the search and study of low-discrepancy sequences. This result states that for any sequence $X=\left\{x_{0}, \ldots, x_{N-1}\right\}$ and any function, $f$, with bounded variation defined on the $s$-dimensional unit cube, $I^{s}$, the integration error over $I^{d}$ is bounded as,

$$
\left|\int_{I^{s}} f(x) d x-\frac{1}{N} \sum_{i=1}^{N} f\left(x_{i}\right)\right| \leq V[f] D_{N}^{*},
$$

where $V[f]$ is the total variation of $f$, in the sense of Hardy-Krause, $D_{N}^{*} 1$ is the star discrepancy of sequence $X$ [20]. As we are normally given a problem to solve, it is only $D_{N}^{*}$ that we can control. This is done by constructing low-discrepancy sequences for use in QMC.

However, the successful parallel implementation of a quasi-Monte Carlo application depends crucially on various quality aspects of the parallel quasirandom sequences used. Randomness can be brought to bear on otherwise deterministic quasirandom sequence by using various scrambling techniques. These methods randomize quasirandom sequences by using pseudorandom numbers to scramble the order the of quasirandom numbers generated or to permute their digits. Thus by the term "scrambling" we are referring more generally to the randomization of quasirandom numbers. Scrambling provides a natural way to parallelize quasirandom sequences, because scrambled quasirandom sequences form a stochastic family which can be assigned to different processes in a parallel computation. In

\footnotetext{
${ }^{1}$ For a sequence of $N$ points $X=\left\{x_{0}, \ldots, x_{N-1}\right\}$ in the $d$-dimensional unit cube $I^{s}$, and for any box, $J$, with one corner at the origin in $I^{s}$, the star discrepancy, $D_{N}^{*}$, is defined as $D_{N}^{*}=\sup _{J \in I^{s}}\left|\mu_{x}(J)-\mu(J)\right|$, where $\mu_{X}(J)=\frac{\text { \#of points in } J}{N}$ is the discrete measure of $J$, i. e., the fraction of points of $X$ in $J$, and $\mu(J)$ is the Lebesgue measure of $J$, i. e., the volume of $J$.
} 
addition, there are many scrambling schemes that produce scrambled sequences with the same $D_{N}^{*}$ as the parent, which means that we expect no degradation of results with these sorts of scrambling. These scrambling schemes are different from other proposed QMC parallelization schemes such as the leap-frog scheme [2] and the blocking scheme [18], which split up a single quasirandom sequence into subsequences.

The Faure sequence is one of the most widely used quasirandom sequences. The original construction of quasirandom sequences was related to the van der Corput sequence, which itself is a one-dimension quasirandom sequence based on digital inversion. This digital inversion method is a central idea behind the construction of many current quasirandom sequences in arbitrary bases and dimensions. Following the construction of the van der Corput sequence, a significant generalization of this method was proposed by Faure [4] to the sequences that now bear his name. In addition, an efficient implementation of the Faure sequence was published shortly afterwards 6]. Later, Tezuka 20] proposed a generalized Faure sequence, GFaure, which forms a family of randomized Faure sequences. We will discuss the scrambling of Faure sequences in this paper.

The organization of this paper is as follows. In $\S 2$, an overview of scrambling methods and a brief introduction to the theory of constructing quasirandom sequences is given. Parallelizations and implementations of quasirandom Faure sequences are presented in $\S 3$. The consequences of choosing a generator matrix, the resulting computational issues, and a some numerical results are illustrated in $\S 4$, and conclusions are presented in $\S 5$.

\section{Scrambling}

Before we begin our discussion of the scrambled Faure sequence, it behooves us to describe, in detail, the standard and widely accepted methods of Faure sequence generation. The reason for this is that construction of Faure sequence follows from the generation of the Van der Corput and Sobol sequences. Often QMC scrambling methods are combined with the original quasirandom number generation algorithms.

The construction of quasirandom sequences is based on finite-field arithmetic. For example, the Sobol sequences [19] are constructed using linear recurring relations over $\mathcal{F}_{2}$. Faure used the Pascal matrix in his construction, and Niederreiter used the formal Laurent series over finite fields to construct low-discrepancy sequences that now bear his name.

We now briefly describe the construction of the above "classical" quasirandom sequences.

- Van der Corput sequences: Let $b \geq 2$ be an integer, and $n$ a non-negative integer with $n=n_{0}+n_{1} b+\ldots+n_{m} b^{m}$ it $b$-adic representation. Then the $n$th term of the Van der Corput sequence is $\phi_{b}(\mathbf{n})=\frac{n_{0}}{b}+\frac{n_{1}}{b^{2}}+\ldots+\frac{n_{m}}{b^{m}}$. Here $\phi_{b}(\mathbf{n})$ is the radical inverse function in base $b$ and and $\mathbf{n}=\left(n_{0}, n_{1}, \ldots, n_{m}\right)^{T}$ 
is the digit vector of the $b$-adic representation of $n . \phi_{b}(\cdot)$ simply reverses the digit expansion of $n$, and places it to the right of the "decimal" point. The Van der Corput sequence in $s$-dimensions, more commonly called the Halton sequence, is one of the most basic one-dimension quasirandom sequences, and can be rewritten in the following form:

$$
\left(\phi_{b_{1}}(\mathbf{C n}), \phi_{b_{2}}(\mathbf{C n}) \ldots, \phi_{b_{s}}(\mathbf{C n})\right)
$$

Here the "generator matrix" $\mathbf{C}$ is the identity matrix and the $n$th Halton sequence is defined as $\left(\phi_{b_{1}}(\mathbf{n}), \phi_{b_{2}}(\mathbf{n}) \ldots, \phi_{b_{s}}(\mathbf{n})\right)$ where the bases, $b_{1}, b_{2}, \ldots, b_{s}$, are pairwise coprime. The other commonly uses quasirandom sequences can be similarly defined by specifying different generator matrices.

- Faure and GFaure sequences: The $n$th element of the Faure sequence is expressed as $\left(\phi_{b}\left(P^{0} \mathbf{n}\right), \phi_{b}\left(P^{1} \mathbf{n}\right), \ldots, \phi_{b}\left(P^{s-1} \mathbf{n}\right)\right)$, where $b$ is prime and $b \geq s$ and $P$ is Pascal matrix whose $(i, j)$ element is equal to $\left(\begin{array}{l}i-1 \\ j-1\end{array}\right)$ Tezuka 22] proposed the generalized Faure sequence, GFaure, with the $j$ th dimension generator matrix $C^{(j)}=A^{(j)} P^{j-1}$, where $A^{(j)}$ are a random nonsingular lower triangular matrices. Faure [5] extended this idea to bigger set. Also a subset of GFaure is called GFaure with the $i$-binomial property [21], where $A^{(j)}$ is defined as:

$$
A^{(j)}=\left(\begin{array}{ccccc}
h_{1} & 0 & 0 & 0 & \ldots \\
g_{2} & h_{1} & 0 & 0 & \ldots \\
g_{3} & g_{2} & h_{1} & 0 & \ldots \\
g_{4} & g_{3} & g_{2} & h_{1} & \ldots \\
\cdot & \cdot & \cdot & \cdot & \ldots \\
\cdot & . & . & \cdot & \ldots \\
\cdot & . & . & . & \ldots
\end{array}\right),
$$

where $h_{1}$ is uniformly distributed on $\mathcal{F}_{b}-\{0\}$ and $g_{i}$ is uniformly distributed on $\mathcal{F}_{b}$. For each $A^{(j)}$, there will be a different random matrix in the above form.

We will generate parallel Faure sequences by randomizing the matrix $A^{(j)}$. However, arbitrary choice of matrix could lead to high correlation between different quasirandom streams. We will address this concern in next section and show how to choose the matrix $A^{(j)}$ so that correlation between streams is minimized.

\section{Parallelization and Implementations}

Much of the recent work on parallelizing quasi-Monte Carlo methods has been aimed at splitting a quasirandom sequence into many subsequences which are then used independently on the various parallel processes. This method works 
well for the parallelization of pseudorandom numbers, but due to the nature of quality in quasirandom numbers, this technique has many drawbacks. In contrast, this paper proposes an alternative approach for generating parallel quasirandom sequences. Here we take a single quasirandom sequence and provide different random digit scramblings of the given sequence. If the scrambling preserves certain equidistribution properties of the parent sequence, then the result will be high-quality quasirandom numbers for each parallel process, and an overall successful parallel quasi-Monte Carlo computation as expected from the Koksma-Hlawka inequality [1].

Parallelization via splitting [18] uses a single quasirandom sequence and assigns subsequences of this quasirandom sequence to different processes. The idea behind splitting is to assume that any subsequence of a given quasirandom sequence has the same uniformity as the parent quasirandom sequence. This is an assumption that is often false 8. In comparison to splitting, each scrambled sequence can be thought of as an independent sequence and thus assigned to a different process, and under certain circumstances it can be proven that the scrambled sequences are as uniform as the parent [15. Since the quality (small discrepancy) of quasirandom sequences is a collective property of the entire sequence, forming new sequences from parts is potentially troublesome. Therefore, scrambled quasirandom sequences provide a very appealing alternative for parallel quasirandom sequences, especially where a single quasirandom sequence is scrambled to provide all the parallel streams. Such a scheme would also be very useful for providing QMC support to the computational Grid [910].

We will consider a variety of generator matrices for parallelizing and implementing parallel Faure sequences. The generation of the original Faure sequences is fast and easy to implement. Here, we applied the same methods as was used in Halton sequences because the original Halton sequence suffers from correlations [3] between radical inverse functions with different bases used for different dimensions. These correlations result in poorly distributed two-dimensional projections. A standard solution to this phenomenon is to use a randomized (scrambled) version of the Halton sequence. We proposed a similar algorithm to improve quality of the Faure sequences. We will use the the same approach here to randomize the generator matrix and thus generate parallel Faure sequences. The strategy used in [3] is to find an optimal $h_{i}$ and $g_{i}$ in base $b$ to improve the quality of the Faure sequence. Most importantly, the two-most significant digits of each Faure point are only scrambled by $h_{1}$ and $g_{2}$. Thus the choice of these two elements is crucial for producing good scrambled Faure sequences. After these choices, the rest of elements in matrix $A^{(j)}$ could be chosen randomly.

Whenever scrambled methods are applied, pseudorandom numbers are the "scramblers". Therefore, it is important to find a good pseudorandom number generator (PRNG) to act as a scrambler so that we can obtain well scrambled quasirandom sequences. A good parallel pseudorandom number generator such as SPRNG [1312] is chosen as our scrambler 2

$\overline{2}$ The SPRNG library can be downloaded at http://sprng.fsu.edu. 


\section{Applications}

FINDER 17, a commercial software system which uses quasirandom sequences to solve problems in finance, is an example of the successful use of GFaure, as a modified GFaure sequence is included in FINDER, where matrices $A^{(j)}$ are empirically chosen to provide high-quality sequences.

Assume that an integrand, $f$, is defined over the $s$-dimensional unit cube, $[0,1)^{s}$, and that $I(f)$ is defined as: $I(f)=\int_{I^{s}} f(x) d x$. Then the $s$-dimensional integral, $I(f)$, in Equation (2) may be approximated by $Q_{N}(f)[14]: Q_{N}(f)=$ $\sum_{i=1}^{N} \omega_{i} f\left(x_{i}\right)$, where $x_{i}$ is in $[0,1)^{s}$, and the $\omega_{i}^{\prime} s$ are weights. If $\left\{x_{1}, \ldots, x_{N}\right\}$ is chosen randomly, and $\omega_{i}=\frac{1}{N}$, then $Q_{N}(f)$ becomes the standard Monte Carlo integral estimate, whose statistical error can be estimated using the Central Limit Theorem. If $\left\{x_{1}, \ldots, x_{N}\right\}$ are a set of quasirandom numbers, then $Q_{N}(f)$ is a quasi-Monte Carlo estimate and the above mentioned Koksma-Hlawka inequality can be appealed to for error bounds.

To empirically test the quality of our proposed parallel Faure sequence, we evaluate the test integral discussed in [6] with $a_{i}=1$

$$
\int_{0}^{1} \ldots \int_{0}^{1} \Pi_{i=1}^{s} \frac{\left|4 x_{i}-2\right|+a_{i}}{1+a_{i}} d x_{1} \ldots d x_{s}=1 .
$$

Table 1. Estimation of The Integral $\int_{0}^{1} \ldots \int_{0}^{1} \Pi_{i=1}^{s} \frac{\left|4 x_{i}-2\right|+1}{2} d x_{1} \ldots d x_{s}=1$

\begin{tabular}{|c|c|c|c|c|c|}
\hline$r$ & $N$ & $s=13$ & $s=20$ & $s=25$ & $s=40$ \\
\hline \hline 10 & 1000 & 0.125 & 0.399 & 0.388 & 0.699 \\
\hline 10 & 3000 & 0.908 & 0.869 & 0.769 & 0.515 \\
\hline 10 & 5000 & 0.912 & 0.985 & 1.979 & 0.419 \\
\hline 10 & 7000 & 0.943 & 1.014 & 1.342 & 0.489 \\
\hline 10 & 30000 & 0.988 & 1.097 & 1.171 & 1.206 \\
\hline 10 & 40000 & 1.014 & 1.118 & 1.181 & 1.118 \\
\hline 10 & 50000 & 1.006 & 1.016 & 1.089 & 1.034 \\
\hline
\end{tabular}

In Table 1, we presented the average of ten $(r=10)$ Faure parallel streams for computing the numerical values of integral. The accuracy of quasi-Monte Carlo integration depends not simply on the dimension of the integrands, but on their effective dimension [23]. It is instructive to publish the results of these numerical integrations when we instead use the original Faure sequence to provide the same number of points as were used in the above computations. Table 2 shows these results. The astonishing fact is that the quality numerical integration using the original is lower than the parallel scrambled Faure sequence. We do not report speedup results, as this is a naturally parallel algorithm, but we feel it important to stress that using scrambling in QMC for parallelization has a very interesting consequence: the quality of the parallel sequences is actually better than the original sequences. The authors are not aware of similar situations, 
Table 2. Estimation of The Integral $\int_{0}^{1} \ldots \int_{0}^{1} \Pi_{i=1}^{s} \frac{\left|4 x_{i}-2\right|+1}{2} d x_{1} \ldots d x_{s}=1$

\begin{tabular}{|c|c|c|c|c|c|}
\hline$r$ & $N$ & $s=13$ & $s=20$ & $s=25$ & $s=40$ \\
\hline \hline 10 & 1000 & 0.412 & 0.456 & 1.300 & 0.677 \\
\hline 10 & 3000 & 1.208 & 0.679 & 0.955 & 0.775 \\
\hline 10 & 5000 & 0.919 & 0.976 & 1.066 & 0.871 \\
\hline 10 & 7000 & 1.043 & 1.214 & 0.958 & 0.916 \\
\hline 10 & 30000 & 0.987 & 1.097 & 0.989 & 1.026 \\
\hline 10 & 40000 & 1.010 & 1.103 & 1.201 & 0.886 \\
\hline 10 & 50000 & 0.996 & 1.006 & 1.178 & 0.791 \\
\hline
\end{tabular}

where parallelization actually increases the quality of a computation, but are interested to learn about more examples in the literature.

\section{Conclusions}

A new scheme for parallel QMC streams using different GFaure sequences is proposed. The advantage of this scheme is that we can provide unlimited independent streams for QMC in heterogeneous computing environment. This scheme is an alternative for generating parallel quasirandom number streams. The obtained are very interesting as the scrambled versions used in individual processes are of higher quality than the original Faure sequence. We need to carry out a big, yet feasible, computations that will provide the data required for a parallel generator based on these ideas. More Numerical experiments, such as application in bioinformatics [1, need to done to further validate this parallel methods. However, this parallelization has a very interesting property. The parallel version of the quasirandom numbers are of better quality than the original sequence. This is due to the fact that the scrambling done for parallelization also increases the quality of the sequences. Thus, not only do we have a faster parallel computation, but the parallel computation is simultaneous more accurate without any extra computational effort.

\section{References}

1. P. Beerli and H. Chi. Quasi-markov chain Monte Carlo to improve inference of population genetic parameters. Mathematics and Computers in Simulation, page In press, 2007.

2. B.C. Bromley. Quasirandom number generators for parallel Monte Carlo algorithms. Journal of Parallel and Distributed Computing, 38(1):101-104, 1996.

3. H. Chi, M. Mascagni, and T. Warnock. On the optimal Halton sequences. Mathematics and Computers in Simulation, 70/1:9-21, 2005.

4. H. Faure. Discrepancy of sequences associated with a number system(in dimension s). Acta. Arith, 41(4):337-351, 1982[French].

5. H. Faure. Variations on(0,s)-sequences. Journal of Complexity, 17(4):741-753, 2001. 
6. B. Fox. Implementation and relative efficiency of quasirandom sequence generators. ACM Trans. on Mathematical Software, 12:362-376, 1986.

7. W. Jank. Efficient simulated maximum likelihood with an application to online retailing. Statistics and Computing, 16:111-124, 2006.

8. L. Kocis and W. Whiten. Computational investigations of low discrepancy sequences. ACM Trans. Mathematical software, 23:266-294, 1997.

9. Y. Li and M. Mascagni. Analysis of large-scale grid-based Monte Carlo applications. International Journal of High Performance Computing Applications, 17(4):369-382, 2003.

10. K. Liu and F. J. Hickernell. A scalable low discrepancy point generator for parallel computing. In Lecture Notes in Computer Science 3358, volume 3358, pages 257 $262,2004$.

11. W. L. Loh. On the asymptotic distribution of scrambled net quadrature. Annals of Statistics, 31:1282-1324, 2003.

12. M. Mascagni and H. Chi. Parallel linear congruential generators with SophieGermain moduli. Parallel Computing, 30:1217-1231, 2004.

13. M. Mascagni and A. Srinivasan. Algorithm 806: SPRNG: A scalable library for pseudorandom number generation. ACM Transactions on Mathematical Software, 26:436-461, 2000.

14. H. Niederreiter. Random number generation and quasi-Monte Carlo methods. SIAM, Philadephia, 1992.

15. A. B. Owen. Randomly permuted $(t, m, s)$-nets and $(t, s)$-sequences. Monte Carlo and Quasi-Monte Carlo Methods in Scientific Computing, 106 in Lecture Notes in Statistics:299-317, 1995.

16. A. B. Owen and S. Tribble. A quasi-Monte Carlo metroplis algorithm. Proceedings of the National Academy of Sciences of the United States of America, 102:88448849, 2005.

17. A. Papageorgiou and J. Traub. Beating Monte Carlo. RISK, 9:63-65, 1997.

18. W. Schmid and A. Uhl. Techniques for parallel quasi-Monte Carlo integration with digital sequences and associated problems. Math. Comput. Simulat, 55(1-3):249257, 2001.

19. I. M. Soboĺ. Uniformly distributed sequences with additional uniformity properties. USSR Comput. Math. and Math. Phy., 16:236-242, 1976.

20. S. Tezuka. Uniform Random Numbers, Theory and Practice. Kluwer Academic Publishers, IBM Japan, 1995.

21. S. Tezuka and H. Faure. I-binomial scrambling of digital nets and sequences. Journal of Complexity, 19(6):744-757, 2003.

22. S. Tezuka and T. Tokuyama. A note on polynomial arithmetic analogue of Halton sequences. ACM Trans. on Modelling and Computer Simulation, 4:279-284, 1994.

23. X. Wang and K.T. Fang. The effective dimension and quasi-monte carlo. Journal of Complexity, 19(2):101-124, 2003. 